\title{
Barriers Of Exclusive Breastfeeding Among Mothers Attending Will-Baby Clinic In Phc, Makkah
}

\author{
1Ahmad Hasan M Alturkstani MD, MPH, 2Ahmed Mahmoud AlSaeed Alahdal MD, 3Sari Ibrahim Asiri MD, SBFM, \\ 4Hossam Hussen Hijazy
}

${ }_{1}$ Public Health Affaire Makkah Region, KSA

2Resident Family Medicine, Ministry of Health, Jeddah, KSA

3General Directorate Assistant of Public Health Affaire Makkah Region, KSA

${ }_{4}$ Family medicine specialist Red Crescent health center, Makkah, KSA

Correspondence Author: Ahmad Hasan M Alturkstani, Public Health Affaire Makkah Region, KSA

E-mail: Hossamoree@gmail.com

Received date: 22 December 2017, Accepted date: 22 January 2018, Online date: 5 February 2018

Copyright: (C) 2018 Ahmad Hasan M Alturkstani et al. This is an open-access article distributed under the terms of the Creative Commons Attribution License, which permits unrestricted use, distribution, and reproduction in any medium, provided the original author and source are credited.

\begin{abstract}
Exclusive breastfeeding is defined as feeding the child nothing but breast milk for the first six months. EBF confers a number of protective benefits for children and mothers. Despite the many benefits of breast feeding it has been shown that there are barriers to the practice of optimal breast feeding. Therefore this study aimed to assess barriers of exclusive breast feeding among breast feeding mothers. A descriptive cross-sectional survey was conducted. Non randomized convenience sampling technique was used to include 150 mothers with healthy infants aged between 0-9 months. Structured interview questionnaire was designed to collect the necessary data. The main study were that the majority of mothers' housewife and multipara and not compliance with exclusive breast feeding. In addition the most common barriers to maintain exclusive breast feeding were the infants still hungry after feeding, perception of insufficient milk production, lack of support from surrounding and return to work. The study concluded that, there were many social constrains that influence mothers' exclusive breast feed their infants. Therefore this study aimed to assess barriers of exclusive breast feeding among breast feeding mothers.
\end{abstract}

Key words: Exclusive breast feeding, barriers, women, infants.

\section{INTRODUCTION}

There is general agreement that breastfeeding is good for the growth and health of infants. The World Health Organization (WHO) recommends exclusive Breast feeding as an important strategy for reducing child deaths, particularly in developing countries. Exclusive breastfeeding is defined as feeding the child nothing but breast milk for the first six months (no foods or liquids including water). After 6 months, breastfeeding is still encouraged, along with the introduction of other foods and liquids. It is also recommended that mothers feed their newborns colostrum, as the first feed immediately after birth. (Froozani, M.D et al., 1999; Otoo. G.E, et al., 2009)

Exclusive breastfeeding confers a number of protective benefits for children and mothers. For example, a longer duration of breastfeeding promotes sensory and cognitive development, protects infants against infectious and chronic diseases, and reduces infant mortality resulting from childhood illnesses such as diarrhea and pneumonia. (American Academy of Pediatrics, 2012; Me Thet M, et al., 2016)

Despite the many benefits of breast feeding it has been shown that there are barriers to the practice of optimal breast feeding. Some of these barriers include hospital practices, advertisement of breast milk substitutes, young mothers, single mothers, lower income and lack of support for the breast feeding mother. Many women identify employment as a barrier to breast feeding. In addition caesarean section and the infant having received supplementation during the first weeks of life. (Mohamed, S.M., et al., 2016) Negative attitudes of women, their partners, family members, health care professionals and the persistently sore and red nipples could be a barrier of breastfeeding and led to early termination. (Piper, S and P. Parks, 2012)

In a study on infant feeding practices among nursing personnel in Australia, returning to work was one of the main reasons women ceased breastfeeding, with 60 percent of women intending to breastfeed when they returned to work, but only 40 percent do so. (Danielle, W., J. Anneka, 2011) It is almost certain that women who go back to work before their babies are six months old will face challenges in adhering to the practice of EBF. (Abigail, A.H., et al., 2013)

Several studies have been conducted to understand the physical, biological, psychological, social, and cultural factors explaining poor lactation and breastfeeding outcomes. (Sibeko, L, et al. Beliefs, 2005; Kakute, P.N., J. Ngum, P. Mitchell, et al., 2005; Cohen, R.J., et al., 1999) Based on the benefits of exclusive breast feeding and continuous reduction in the number of breastfed mothers, the investigator found it necessary to explore the barriers to exclusive breast feeding among breastfed women.

Aim of the study:

The aim of this study was to assess barriers of exclusive breast feeding among breast feeding mothers.

Research question:

What are the most common barriers of exclusive breastfeeding among breast feeding mothers?

Study design:

A descriptive cross-sectional survey was conducted in carrying out of this study. 
Citation: Ahmad Hasan M Alturkstani MD, MPH, Ahmed Mahmoud AlSaeed Alahdal MD, Sari Ibrahim Asiri MD, SBFM, Hossam Hussen Hijazy,2018

Barriers Of Exclusive Breastfeeding Among Mothers Attending Will -Baby Clinic In Phc, Makkah. Australian Journal of Basic and Applied Sciences., 12(1):

54-61.

Study setting:

the study was conducted at mother child clinic at primary health care centers at Makah.

Study population:

non randomized convenience sampling technique was used to include 150 mothers with healthy infants aged between 0-9 months and willing to participate in the study. Mothers with infants who were preterm, twins and birth defects were excluded from the study.

Tools of the study:

Structured interview questionnaire was designed to collect the necessary data, and developed by the researchers after review of the literature and reviewed by experts and modified after application of pilot study. It consisted of:

Part one:

Mothers sociodemographic characteristics: this part consisted data about mother's age, age at marriage, living with extended family, level of education, occupation, parity and gender of baby.

Part two:

included data about sources and factors that influencing the mother's preference for breastfeeding as social norm, helping baby to grow in a normal pattern, providing baby with natural immunity, a form of child spacing, easy and comfortable and returned body to normal.

Part three:

this part explain the reasons for breast feeding preference as Encouragement from mother, husband, nurse, mother-law, neighbours or members of my religious affiliation, Social pressure on mothers and their personal determination/experience.

\section{Part four:}

include items that determine the constraints and breastfeeding challenges which are maternal problems, feeling of pain or discomfort, poor growing baby or still hungry, or pressure from surrounding and return to work.

Method:

A pilot study:

was carried out before starting of data collection on (10\%) of the study period for the purpose to test the clarity, completeness, and to determine the time involvement. According to the results of the pilot study, the needed modification, omissions, and/or additions were done.

Data collection:

was done by the researcher from $1^{\text {st }}$ October to 30 November The researcher interviewed each participated woman individually to obtain the necessary information. The actual work started by meeting the woman, the researcher firstly introduced self to them and gave them a complete back ground about the study. The researcher gave mother (who can read and write), the sheet which was pre-designed in Arabic language and stay with them to clarified any question vague to them or to read the sheet if the mother unable to read it. The sheet required about 15-20 minutes for filling it.

\section{Statistical analysis:}

Data were analyzed using the statistical package for social science (SPSS) version 20.0 (Windows Microsoft). Data were presented using descriptive statistics in the form of frequencies and percentages for qualitative variables, means and standard deviations for quantitative variables. Quantitative continuous data were compared using Chi. Square to determine significance for non-parametric variable. Probability (p-value) less than 0.05 was considered significant.

\section{Ethical consideration:}

Administrative approval was obtained from the responsible persons. The mothers have ethical rights to agree or refuse to participate in the study. Consent to participate in the study was secured orally and informed that the information obtained will be confidential and used for the purpose of the study.

Results:

This table shows that more than two fifth (41.3\%) were aged from 30-34 years followed by $24 \%$ aged from 20-24 years of age. Regarding age at marriage, more than half of women were married at 20-25 years of age and more than one quarter (26\%) marry before 20 years of age. As regards level of education, the highest percentages $(41.3 \%, 24.7 \%)$ were for secondary and university education respectively. The majority of women $(90.7 \%)$ were house wives and two thirds $(62 \%)$ of them delivered at governmental hospitals. Concerning parity, the majority (82\%) of women was multiparous and more than half of them (59.3\%) had a planned pregnancy. Approximately half of women has female baby with less than three months age.as for the age of administering food rather than breast, this table revealed that two thirds (68\%) of women administer food at 3-6 months of baby age.

Table I: Frequency distribution of women sociodemographic characteristics:

\begin{tabular}{|c|c|c|}
\hline Sociodemographic characteristics & $\mathrm{N}$ & $\%$ \\
\hline \multicolumn{3}{|l|}{ Age: } \\
\hline $20-24$ & 36 & 24.0 \\
\hline $25-29$ & 20 & 13.3 \\
\hline $30-34$ & 62 & 41.3 \\
\hline $35-39$ & 32 & 21.3 \\
\hline \multicolumn{3}{|l|}{ Age at marriage (years) } \\
\hline$<20$ & 39 & 26.0 \\
\hline $20-25$. & 78 & 52.0 \\
\hline$>25$ & 33 & 22.0 \\
\hline \multicolumn{3}{|l|}{ Women living with extended families } \\
\hline Yes & 24 & 16.0 \\
\hline No & 126 & 84.0 \\
\hline \multicolumn{3}{|l|}{ Level of education } \\
\hline Illiterate & 15 & 10.0 \\
\hline Primary & 21 & 14.0 \\
\hline Secondary & 62 & 41.3 \\
\hline University & 52 & 34.7 \\
\hline \multicolumn{3}{|l|}{ Occupation: } \\
\hline Yes & 14 & 9.3 \\
\hline
\end{tabular}


Citation: Ahmad Hasan M Alturkstani MD, MPH, Ahmed Mahmoud AlSaeed Alahdal MD, Sari Ibrahim Asiri MD, SBFM, Hossam Hussen Hijazy,2018

Barriers Of Exclusive Breastfeeding Among Mothers Attending Will -Baby Clinic In Phc, Makkah. Australian Journal of Basic and Applied Sciences., 12(1):

54-61.

\begin{tabular}{|c|c|c|}
\hline No & 136 & 90.7 \\
\hline \multicolumn{3}{|l|}{ Place of delivery } \\
\hline Governmental hospital & 93 & 62.0 \\
\hline Private hospital & 54 & 36.0 \\
\hline At home & 3 & 2.0 \\
\hline \multicolumn{3}{|l|}{ Parity: } \\
\hline Primiparous & 27 & 18.0 \\
\hline Multiparous & 123 & 82.0 \\
\hline \multicolumn{3}{|l|}{ Planned pregnancy } \\
\hline Yes & 89 & 59.3 \\
\hline No & 61 & 40.7 \\
\hline \multicolumn{3}{|l|}{ Gender of baby } \\
\hline Male & 72 & 48.0 \\
\hline Female & 78 & 52.0 \\
\hline \multicolumn{3}{|l|}{ Age of baby: } \\
\hline$<3$ months & 72 & 48.0 \\
\hline $3-6$ months & 42 & 28.0 \\
\hline $7-10$ months & 12 & 8.0 \\
\hline 11 to 12 months & 24 & 16.0 \\
\hline \multicolumn{3}{|c|}{ Age of administering food rather than breast } \\
\hline Not eat & 48 & 32.0 \\
\hline 3-6 months & 102 & 68.0 \\
\hline
\end{tabular}

Table II: Frequency distributions of factors influencing preference of breast feeding:

This table revealed that the highest percentages of women $(100 \%, 94.7 \%, 57.3 \%)$ preferred breast feeding because it helps the baby to grow in a normal pattern, provides baby with natural immunity and returns the mother's body to normal respectively.

Table II: Frequency distributions of factors influencing preference of breast feeding:

\begin{tabular}{|c|c|c|c|c|c|c|c|}
\hline \multirow{3}{*}{\multicolumn{2}{|c|}{ Items }} & \multicolumn{4}{|c|}{ Factors influencing preference for breastfeeding } & \multirow{2}{*}{\multicolumn{2}{|c|}{ Chi-square }} \\
\hline & & \multicolumn{2}{|c|}{ Yes } & \multicolumn{2}{|c|}{ No } & & \\
\hline & & $\mathrm{N}$ & $\%$ & $\mathrm{~N}$ & $\%$ & \multirow{2}{*}{$\begin{array}{l}X^{2} \\
1.307\end{array}$} & \multirow{2}{*}{$\begin{array}{l}\text { P-value } \\
0.253\end{array}$} \\
\hline 1 & Social norm as a mother & 68 & $45.3 \%$ & 82 & $54.7 \%$ & & \\
\hline 2 & Helping baby to grow in a normal pattern & 150 & $100.0 \%$ & 0 & $0.0 \%$ & - & - \\
\hline 3 & Providing baby with natural immunity & 142 & $94.7 \%$ & 8 & $5.3 \%$ & 119.707 & 0.000 \\
\hline 4 & Is a form of child spacing & 18 & $12.0 \%$ & 132 & $88.0 \%$ & 86.640 & 0.000 \\
\hline 5 & Easy and comfortable & 26 & $17.3 \%$ & 124 & $82.7 \%$ & 64.027 & 0.000 \\
\hline 6 & Returned body to normal & 86 & $57.3 \%$ & 64 & $42.7 \%$ & 3.227 & 0.072 \\
\hline
\end{tabular}

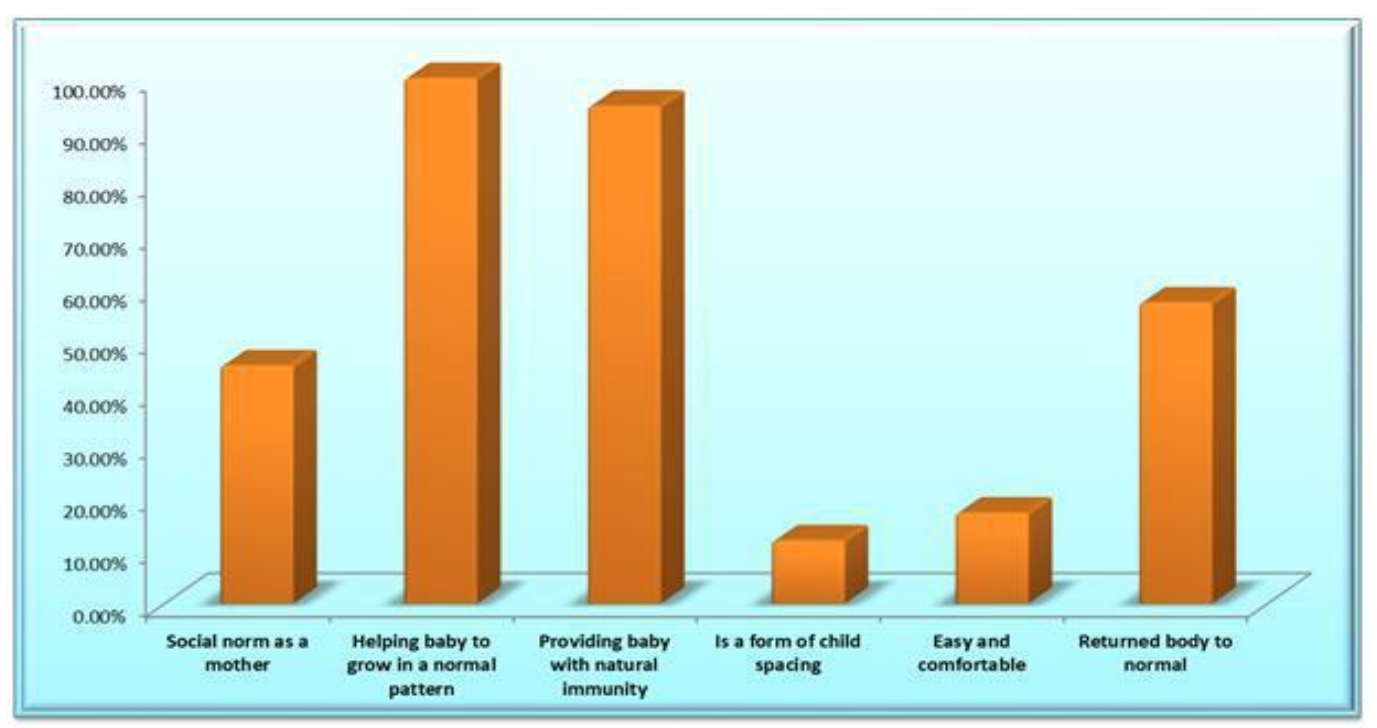

Table III: Frequency distribution of reasons for breast feeding preference:

It was observed that factors such as women personal determination/experience (96\%), encouragement from husband (92.7\%), mother (88\%)and mother-law $(66.7 \%)$ take the highest percentages. 
Citation: Ahmad Hasan M Alturkstani MD, MPH, Ahmed Mahmoud AlSaeed Alahdal MD, Sari Ibrahim Asiri MD, SBFM, Hossam Hussen Hijazy,2018

Barriers Of Exclusive Breastfeeding Among Mothers Attending Will -Baby Clinic In Phc, Makkah. Australian Journal of Basic and Applied Sciences., 12(1):

54-61.

Table III: Frequency distribution of reasons for breast feeding preference:

\begin{tabular}{l}
\hline \multirow{2}{*}{ Items } \\
\cline { 3 - 7 }
\end{tabular}

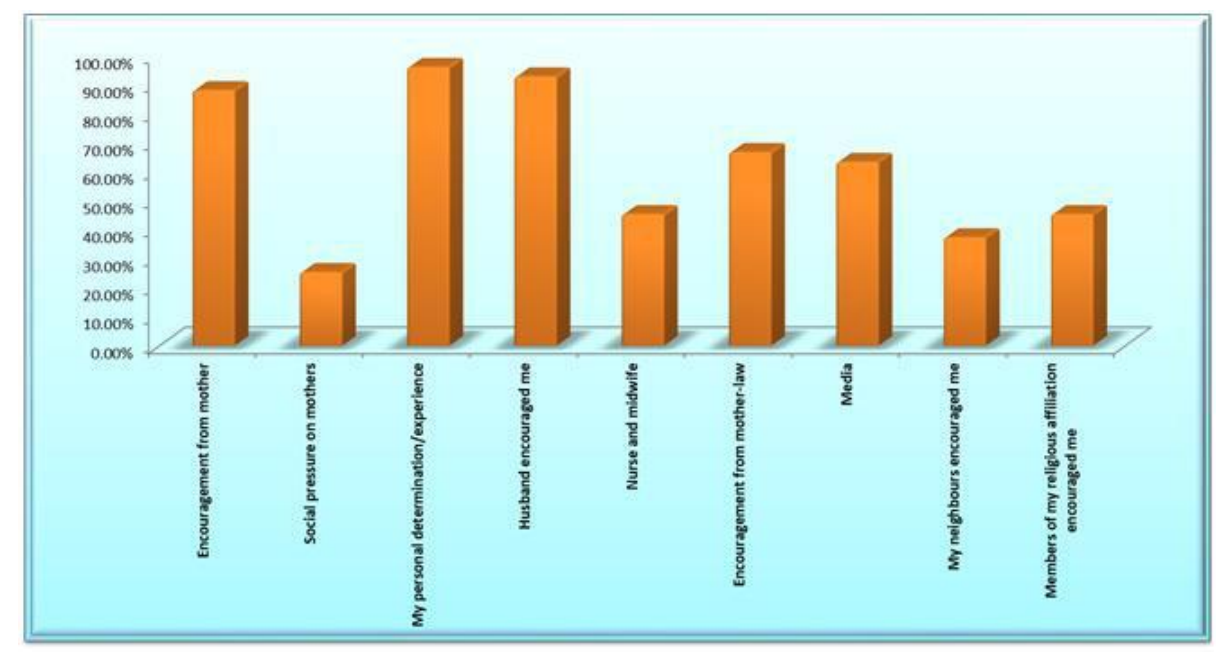

Table IV: Frequency distribution of the constrains and breast feeding challenges:

This table shows that presence of insufficient breast milk to satisfy the baby (80\%), continue to be hungry after eating $(68 \%)$, and the baby doesn't gain enough weight $(64.7 \%)$ are the factors that take the highest frequencies.

Table IV: Frequency distribution of the constrains and breast feeding challenges:

\begin{tabular}{|c|c|c|c|c|c|c|c|}
\hline \multirow{3}{*}{\multicolumn{2}{|c|}{ Items }} & \multicolumn{4}{|c|}{ Constraints and breastfeeding challenges } & \multirow{2}{*}{\multicolumn{2}{|c|}{ Chi-square }} \\
\hline & & \multicolumn{2}{|c|}{ Yes } & \multicolumn{2}{|c|}{ No } & & \\
\hline & & $\mathrm{N}$ & $\%$ & $\mathrm{~N}$ & $\%$ & $\mathrm{X}^{2}$ & P-value \\
\hline 1 & Baby continued to be hungry after feeding & 102 & $68.0 \%$ & 48 & $32.0 \%$ & 19.440 & 0.000 \\
\hline 2 & Maternal health problem & 11 & $7.3 \%$ & 139 & $92.7 \%$ & 109.227 & 0.000 \\
\hline 3 & Fear of infant becoming addicted to breast milk & 33 & $22.0 \%$ & 117 & $78.0 \%$ & 47.040 & 0.000 \\
\hline 4 & Due to pains in my breast & 42 & $28.0 \%$ & 108 & $72.0 \%$ & 29.040 & 0.000 \\
\hline 5 & My mother-law pressured me to wean the baby & 0 & $0.0 \%$ & 150 & $100.0 \%$ & - & - \\
\hline 6 & Was not making enough breast milk to satisfy my child & 120 & $80.0 \%$ & 30 & $20.0 \%$ & 54.000 & 0.000 \\
\hline 7 & I returned to work/business & 14 & $9.3 \%$ & 136 & $90.7 \%$ & 99.227 & 0.000 \\
\hline 8 & Lack of husband's support & 17 & $11.3 \%$ & 133 & $88.7 \%$ & 89.707 & 0.000 \\
\hline 9 & Breastfeeding was too tiring & 47 & $31.3 \%$ & 103 & $68.7 \%$ & 20.907 & 0.000 \\
\hline 10 & My neighbours pressured me to wean the baby & 6 & $4.0 \%$ & 144 & $96.0 \%$ & 126.960 & 0.000 \\
\hline 11 & Baby refused breast milk & 39 & $26.0 \%$ & 111 & $74.0 \%$ & 34.560 & 0.000 \\
\hline 12 & Was losing weight & 70 & $46.7 \%$ & 80 & $53.3 \%$ & 0.667 & 0.414 \\
\hline 13 & I feel dizzy at times during breastfeeding & 52 & $34.7 \%$ & 98 & $65.3 \%$ & 14.107 & 0.000 \\
\hline 14 & Due to pregnancy & 14 & $9.3 \%$ & 136 & $90.7 \%$ & 99.227 & 0.000 \\
\hline 15 & My baby was not gaining enough weight & 97 & $64.7 \%$ & 53 & $35.3 \%$ & 12.907 & 0.000 \\
\hline 16 & I was not feeding well & 88 & $58.7 \%$ & 62 & $41.3 \%$ & 4.507 & 0.034 \\
\hline
\end{tabular}


Citation: Ahmad Hasan M Alturkstani MD, MPH, Ahmed Mahmoud AlSaeed Alahdal MD, Sari Ibrahim Asiri MD, SBFM, Hossam Hussen Hijazy,2018 Barriers Of Exclusive Breastfeeding Among Mothers Attending Will -Baby Clinic In Phc, Makkah. Australian Journal of Basic and Applied Sciences., 12(1): 54-61.

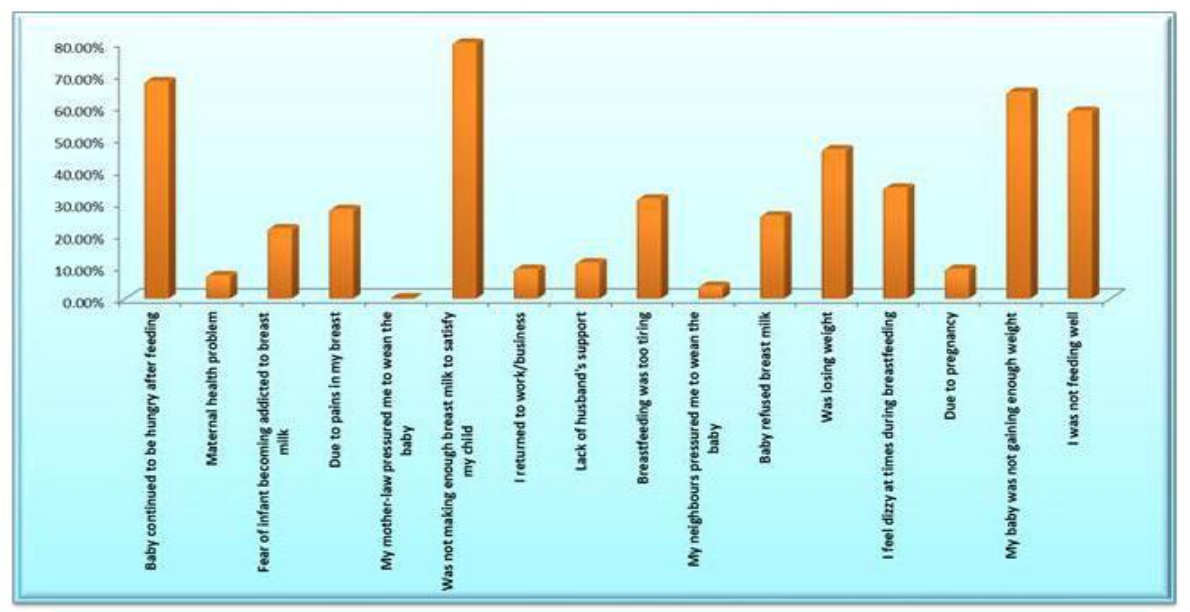

Table V: significant difference between factors affecting EBF and age at marriage and living with extended family:

Significance of difference between barriers of exclusive breast feeding and age of marriage, living in extended family: this table shows a statistical significant difference between the women age at marriage and factors influencing exclusive breast feeding as providing baby with natural immunity, form of child spacing, return body to normal, women personal experience, encouragement from husband and neighbors. Regarding barriers there were a a statistical significant difference between the women age at marriage and baby continuing hungry, maternal health problems, return to work and lack of husband support.

Table V: significant difference between factors affecting EBF and age at marriage and living with extended family:

\begin{tabular}{|c|c|c|c|c|c|c|c|c|c|c|c|c|}
\hline & \multicolumn{6}{|c|}{ Age at marriage (years) } & \multirow{3}{*}{$\begin{array}{l}\mathrm{P}- \\
\text { value }\end{array}$} & \multicolumn{4}{|c|}{$\begin{array}{l}\text { Women living with extended } \\
\text { families }\end{array}$} & \multirow{3}{*}{ P-value } \\
\hline & \multicolumn{2}{|c|}{$<20$} & \multicolumn{2}{|c|}{$20-25$} & \multicolumn{2}{|c|}{$>25$} & & \multicolumn{2}{|c|}{ yes } & \multicolumn{2}{|l|}{ No } & \\
\hline & $\mathrm{N}$ & $\%$ & $\mathrm{~N}$ & $\%$ & $\mathrm{~N}$ & $\%$ & & $\mathrm{~N}$ & $\%$ & $\mathrm{~N}$ & $\%$ & \\
\hline Social norm as a mother & 12 & $17.6 \%$ & 41 & $60.3 \%$ & 15 & $22.1 \%$ & 0.078 & 12 & $17.6 \%$ & 56 & $82.4 \%$ & 0.617 \\
\hline Helping baby to grow in a normal pattern & 39 & $26.0 \%$ & 78 & $52.0 \%$ & 33 & $22.0 \%$ & 1.000 & 24 & $16.0 \%$ & 126 & $84.0 \%$ & 1.000 \\
\hline Providing baby with natural immunity & 39 & $27.5 \%$ & 70 & $49.3 \%$ & 33 & $23.2 \%$ & $0.004 *$ & 24 & $16.9 \%$ & 118 & $83.1 \%$ & 0.090 \\
\hline Is a form of child spacing & 6 & $33.3 \%$ & 12 & $66.7 \%$ & 0 & $0.0 \%$ & $0.008^{*}$ & 6 & $33.3 \%$ & 12 & $66.7 \%$ & $0.05^{*}$ \\
\hline Easy and comfortable & 3 & $11.5 \%$ & 14 & $53.8 \%$ & 9 & $34.6 \%$ & 0.078 & 3 & $11.5 \%$ & 23 & $88.5 \%$ & 0.480 \\
\hline Returned body to normal & 27 & $31.4 \%$ & 35 & $40.7 \%$ & 24 & $27.9 \%$ & $0.005^{*}$ & 9 & $10.5 \%$ & 77 & $89.5 \%$ & $0.033 *$ \\
\hline Encouragement from mother & 33 & $25.0 \%$ & 69 & $52.3 \%$ & 30 & $22.7 \%$ & 0.707 & 24 & $18.2 \%$ & 108 & $81.8 \%$ & $0.009 *$ \\
\hline Social pressure on mothers & 9 & $23.7 \%$ & 20 & $52.6 \%$ & 9 & $23.7 \%$ & 0.916 & 0 & $0.0 \%$ & 38 & $100.0 \%$ & $0.000 *$ \\
\hline My personal determinatior/experience & 33 & $22.9 \%$ & 78 & $54.2 \%$ & 33 & $22.9 \%$ & $0.000^{*}$ & 18 & $12.5 \%$ & 126 & $87.5 \%$ & $0.000 *$ \\
\hline Husband encouraged me & 36 & $25.9 \%$ & 76 & $54.7 \%$ & 27 & $19.4 \%$ & $0.022 *$ & 24 & $17.3 \%$ & 115 & $82.7 \%$ & $0.046^{*}$ \\
\hline Nurse and midwife & 18 & $26.5 \%$ & 38 & $55.9 \%$ & 12 & $17.6 \%$ & 0.482 & 6 & $8.8 \%$ & 62 & $91.2 \%$ & $0.025 *$ \\
\hline Encouragement from mother-law & 30 & $30.0 \%$ & 49 & $49.0 \%$ & 21 & $21.0 \%$ & 0.271 & 18 & $18.0 \%$ & 82 & $82.0 \%$ & 0.335 \\
\hline Media & 21 & $22.1 \%$ & 50 & $52.6 \%$ & 24 & $25.3 \%$ & 0.247 & 9 & $9.5 \%$ & 86 & $90.5 \%$ & $0.005 *$ \\
\hline My neighbours encouraged me & 15 & $26.8 \%$ & 23 & $41.1 \%$ & 18 & $32.1 \%$ & $0.046^{*}$ & 9 & $16.1 \%$ & 47 & $83.9 \%$ & 0.985 \\
\hline $\begin{array}{l}\text { Members of my religious affiliation } \\
\text { encouraged me }\end{array}$ & 15 & $22.1 \%$ & 35 & $51.5 \%$ & 18 & $26.5 \%$ & 0.390 & 9 & $13.2 \%$ & 59 & $86.8 \%$ & 0.398 \\
\hline Baby continued to be hungry after feeding & 21 & $20.6 \%$ & 63 & $61.8 \%$ & 18 & $17.6 \%$ & $0.002 *$ & 0 & $0.0 \%$ & 102 & $100.0 \%$ & $0.000 *$ \\
\hline Matemal health problem & 9 & $81.8 \%$ & 2 & $18.2 \%$ & 0 & $0.0 \%$ & $0.000^{*}$ & 3 & $27.3 \%$ & 8 & $72.7 \%$ & 0.323 \\
\hline $\begin{array}{l}\text { Fear of infant becoming addicted to breast } \\
\text { milk }\end{array}$ & 9 & $27.3 \%$ & 18 & $54.5 \%$ & 6 & $18.2 \%$ & 0.831 & 3 & $9.1 \%$ & 30 & $90.9 \%$ & 0.196 \\
\hline Due to pains in my breast & 21 & $50.0 \%$ & 18 & $42.9 \%$ & 3 & $7.1 \%$ & $0.000 *$ & 3 & $7.1 \%$ & 39 & $92.9 \%$ & $0.049 *$ \\
\hline $\begin{array}{l}\text { was not making enough breast milk to } \\
\text { satisfy my child }\end{array}$ & 27 & $22.5 \%$ & 66 & $55.0 \%$ & 27 & $22.5 \%$ & 0.157 & 15 & $12.5 \%$ & 105 & $87.5 \%$ & $0.028 *$ \\
\hline I retumed to work/business & 3 & $21.4 \%$ & 11 & $78.6 \%$ & 0 & $0.0 \%$ & $0.015^{*}$ & 0 & $0.0 \%$ & 14 & $100.0 \%$ & $0.023 *$ \\
\hline Lack of husband's support & 9 & $52.9 \%$ & 2 & $11.8 \%$ & 6 & $35.3 \%$ & $0.001^{*}$ & 3 & $17.6 \%$ & 14 & $82.4 \%$ & 0.846 \\
\hline Breastfeeding was too tiring & 12 & $25.5 \%$ & 29 & $61.7 \%$ & 6 & $12.8 \%$ & 0.126 & 3 & $6.4 \%$ & 44 & $93.6 \%$ & $0.02 *$ \\
\hline $\begin{array}{l}\text { My neighbours pressured me to wean the } \\
\text { baby }\end{array}$ & 0 & $0.0 \%$ & 3 & $50.0 \%$ & 3 & $50.0 \%$ & 0.089 & 6 & $100.0 \%$ & 0 & $0.0 \%$ & $0.000^{*}$ \\
\hline Baby refused breast milk & 6 & $15.4 \%$ & 27 & $69.2 \%$ & 6 & $15.4 \%$ & $0.039^{*}$ & 9 & $23.1 \%$ & 30 & $76.9 \%$ & 0.174 \\
\hline was losing weight & 30 & $42.9 \%$ & 28 & $40.0 \%$ & 12 & $17.1 \%$ & $0.000^{*}$ & 12 & $17.1 \%$ & 58 & $82.9 \%$ & 0.721 \\
\hline I feel dizzy at times during breastfeeding & 12 & $23.1 \%$ & 28 & $53.8 \%$ & 12 & $23.1 \%$ & 0.835 & 3 & $5.8 \%$ & 49 & $94.2 \%$ & $0.008 *$ \\
\hline Due to pregnancy & 3 & $21.4 \%$ & 8 & $57.1 \%$ & 3 & $21.4 \%$ & 0.900 & 6 & $42.9 \%$ & 8 & $57.1 \%$ & $0.011 *$ \\
\hline My baby was not gaining enough weight & 15 & $15.5 \%$ & 58 & $59.8 \%$ & 24 & $24.7 \%$ & $0.000^{*}$ & 9 & $9.3 \%$ & 88 & $90.7 \%$ & $0.003 *$ \\
\hline I was not feeding well & 15 & $17.0 \%$ & 55 & $62.5 \%$ & 18 & $20.5 \%$ & $0.003^{*}$ & 12 & $13.6 \%$ & 76 & $86.4 \%$ & 0.350 \\
\hline
\end{tabular}

Table VI: Significance of difference between factors affecting exclusive breast feeding and level of education:

This table revealed a statistical significant difference between level of education and mother's social norms, form of child spacing, return body to normal, social pressure, personal determination,media and the majority of barriers affecting exclusive breast feeding. 
Citation: Ahmad Hasan M Alturkstani MD, MPH, Ahmed Mahmoud AlSaeed Alahdal MD, Sari Ibrahim Asiri MD, SBFM, Hossam Hussen Hijazy,2018 Barriers Of Exclusive Breastfeeding Among Mothers Attending Will -Baby Clinic In Phc, Makkah. Australian Journal of Basic and Applied Sciences., 12(1): 54-61.

Table VI: Significance of difference between factors affecting exclusive breast feeding and level of education:

\begin{tabular}{|c|c|c|c|c|c|c|c|c|c|}
\hline & \multicolumn{8}{|c|}{ Level of education } & \multirow{3}{*}{ P-value } \\
\hline & \multicolumn{2}{|c|}{ Illiterate } & \multicolumn{2}{|c|}{ Primary } & \multicolumn{2}{|c|}{ Secondary } & \multicolumn{2}{|c|}{ University } & \\
\hline & $\mathrm{N}$ & $\%$ & $\mathrm{~N}$ & $\%$ & $\mathrm{~N}$ & $\%$ & $\mathrm{~N}$ & $\%$ & \\
\hline Social norm as a mother & 9 & $13.2 \%$ & 3 & $4.4 \%$ & 33 & $48.5 \%$ & 23 & $33.8 \%$ & $0.007 *$ \\
\hline Helping baby to grow in a normal pattern & 15 & $10.0 \%$ & 21 & $14.0 \%$ & 62 & $41.3 \%$ & 52 & $34.7 \%$ & 1.000 \\
\hline Providing baby with natural immunity & 15 & $10.6 \%$ & 21 & $14.8 \%$ & 56 & $39.4 \%$ & 50 & $35.2 \%$ & 0.107 \\
\hline Is a form of child spacing & 0 & $0.0 \%$ & 9 & $50.0 \%$ & 6 & $33.3 \%$ & 3 & $16.7 \%$ & $0.000^{*}$ \\
\hline Easy and comfortable & 0 & $0.0 \%$ & 3 & $11.5 \%$ & 6 & $23.1 \%$ & 17 & $65.4 \%$ & $0.001^{*}$ \\
\hline Returned body to normal & 6 & $7.0 \%$ & 6 & $7.0 \%$ & 38 & $44.2 \%$ & 36 & $41.9 \%$ & $0.006^{*}$ \\
\hline Encouragement from mother & 15 & $11.4 \%$ & 18 & $13.6 \%$ & 53 & $40.2 \%$ & 46 & $34.8 \%$ & 0.231 \\
\hline Social pressure on mothers & 0 & $0.0 \%$ & 6 & $15.8 \%$ & 24 & $63.2 \%$ & 8 & $21.1 \%$ & $0.001^{*}$ \\
\hline My personal determinatior/experience & 9 & $6.3 \%$ & 21 & $14.6 \%$ & 62 & $43.1 \%$ & 52 & $36.1 \%$ & $0.000^{*}$ \\
\hline Husband encouraged me & 15 & $10.8 \%$ & 21 & $15.1 \%$ & 56 & $40.3 \%$ & 47 & $33.8 \%$ & 0.098 \\
\hline Nurse and midwife & 6 & $8.8 \%$ & 9 & $13.2 \%$ & 27 & $39.7 \%$ & 26 & $38.2 \%$ & 0.859 \\
\hline Encouragement from mother-law & 12 & $12.0 \%$ & 12 & $12.0 \%$ & 39 & $39.0 \%$ & 37 & $37.0 \%$ & 0.391 \\
\hline Media & 9 & $9.5 \%$ & 6 & $6.3 \%$ & 51 & $53.7 \%$ & 29 & $30.5 \%$ & $0.000^{*}$ \\
\hline My neighbours encouraged me & 6 & $10.7 \%$ & 3 & $5.4 \%$ & 36 & $64.3 \%$ & 11 & $19.6 \%$ & $0.000^{*}$ \\
\hline Members of my religious affiliation encouraged me & 6 & $8.8 \%$ & 6 & $8.8 \%$ & 36 & $52.9 \%$ & 20 & $29.4 \%$ & 0.053 \\
\hline Baby continued to be hungry after feeding & 0 & $0.0 \%$ & 12 & $11.8 \%$ & 53 & $52.0 \%$ & 37 & $36.3 \%$ & $0.000^{*}$ \\
\hline Matemal health problem & 0 & $0.0 \%$ & 3 & $27.3 \%$ & 0 & $0.0 \%$ & 8 & $72.7 \%$ & $0.001^{*}$ \\
\hline Fear of infant becoming addicted to breast milk & 0 & $0.0 \%$ & 3 & $9.1 \%$ & 24 & $72.7 \%$ & 6 & $18.2 \%$ & $0.000^{*}$ \\
\hline Due to pains in my breast & 6 & $14.3 \%$ & 3 & $7.1 \%$ & 18 & $42.9 \%$ & 15 & $35.7 \%$ & 0.349 \\
\hline was not making enough breast milk to satisf my child & 6 & $5.0 \%$ & 18 & $15.0 \%$ & 59 & $49.2 \%$ & 37 & $30.8 \%$ & $0.000^{*}$ \\
\hline I retumed to work/business & 0 & $0.0 \%$ & 0 & $0.0 \%$ & 0 & $0.0 \%$ & 14 & $100.0 \%$ & $0.000^{*}$ \\
\hline Lack ofhusband's support & 0 & $0.0 \%$ & 3 & $17.6 \%$ & 12 & $70.6 \%$ & 2 & $11.8 \%$ & $0.012^{*}$ \\
\hline Breastfeeding was too tiring & 6 & $12.8 \%$ & 3 & $6.4 \%$ & 21 & $44.7 \%$ & 17 & $36.2 \%$ & 0.261 \\
\hline My neighbours pressured me to wean the baby & 0 & $0.0 \%$ & 3 & $50.0 \%$ & 0 & $0.0 \%$ & 3 & $50.0 \%$ & $0.017^{*}$ \\
\hline Baby refused breast milk & 3 & $7.7 \%$ & 6 & $15.4 \%$ & 12 & $30.8 \%$ & 18 & $46.2 \%$ & 0.287 \\
\hline was losing weight & 9 & $12.9 \%$ & 3 & $4.3 \%$ & 41 & $58.6 \%$ & 17 & $24.3 \%$ & $0.000^{*}$ \\
\hline I feel dizzy at times during breastfeeding & 6 & $11.5 \%$ & 3 & $5.8 \%$ & 27 & $51.9 \%$ & 16 & $30.8 \%$ & 0.069 \\
\hline Due to pregnancy & 0 & $0.0 \%$ & 3 & $21.4 \%$ & 0 & $0.0 \%$ & 11 & $78.6 \%$ & $0.000^{*}$ \\
\hline My baby was not gaining enough weight & 0 & $0.0 \%$ & 18 & $18.6 \%$ & 44 & $45.4 \%$ & 35 & $36.1 \%$ & $0.000^{*}$ \\
\hline I was not feeding well & 0 & $0.0 \%$ & 18 & $20.5 \%$ & 38 & $43.2 \%$ & 32 & $36.4 \%$ & $0.000^{*}$ \\
\hline
\end{tabular}

Table VII: Significance of difference between factors affecting exclusive breast feeding with occupation and age of baby:

This table showed that there were a statistical significant difference between the occupation, age of baby and factors affecting exclusive breast feeding.

Table VII: Significance of difference between factors affecting exclusive breast feeding with occupation and age of baby:

\begin{tabular}{|c|c|c|c|c|c|c|c|c|c|c|c|c|c|c|}
\hline & \multicolumn{4}{|c|}{ Occupation } & \multirow{3}{*}{$\begin{array}{l}\mathrm{P}- \\
\text { value }\end{array}$} & \multicolumn{8}{|c|}{ Age of baby: } & \multirow{3}{*}{$\begin{array}{l}\mathrm{P}- \\
\text { value }\end{array}$} \\
\hline & \multicolumn{2}{|c|}{ yes } & \multicolumn{2}{|l|}{ No } & & \multicolumn{2}{|c|}{$\begin{array}{l}\text { Less than } 3 \\
\text { months }\end{array}$} & \multicolumn{2}{|c|}{ 3-6 months } & \multicolumn{2}{|c|}{ 7-10 months } & \multicolumn{2}{|c|}{$\begin{array}{l}11 \text { to } 12 \\
\text { months }\end{array}$} & \\
\hline & $\mathrm{N}$ & $\%$ & $\mathrm{~N}$ & $\%$ & & $\mathrm{~N}$ & $\%$ & $\mathrm{~N}$ & $\%$ & $\mathrm{~N}$ & $\%$ & $\mathrm{~N}$ & $\%$ & \\
\hline Social norm as a mother & 5 & $7.4 \%$ & 63 & $92.6 \%$ & 0.444 & 39 & $57.4 \%$ & 15 & $22.1 \%$ & 12 & $17.6 \%$ & 2 & $2.9 \%$ & $0.000^{*}$ \\
\hline $\begin{array}{l}\text { Helping baby to grow in a } \\
\text { normal pattern }\end{array}$ & 14 & $9.3 \%$ & 136 & $90.7 \%$ & 1.000 & 72 & $48.0 \%$ & 42 & $28.0 \%$ & 12 & $8.0 \%$ & 24 & $16.0 \%$ & 1.000 \\
\hline $\begin{array}{l}\text { Providing baby with natural } \\
\text { immunity }\end{array}$ & 12 & $8.5 \%$ & 130 & $91.5 \%$ & 0.180 & 72 & $50.7 \%$ & 36 & $25.4 \%$ & 12 & $8.5 \%$ & 22 & $15.5 \%$ & $0.003 *$ \\
\hline Is a form of child spacing & 0 & $0.0 \%$ & 18 & $100.0 \%$ & 0.052 & 12 & $66.7 \%$ & 3 & $16.7 \%$ & 0 & $0.0 \%$ & 3 & $16.7 \%$ & 0.139 \\
\hline Easy and comfortable & 5 & $19.2 \%$ & 21 & $80.8 \%$ & 0.081 & 21 & $80.8 \%$ & 0 & $0.0 \%$ & 3 & $11.5 \%$ & 2 & $7.7 \%$ & $0.000^{*}$ \\
\hline Returned body to normal & 12 & $14.0 \%$ & 74 & $86.0 \%$ & $0.017 *$ & 42 & $48.8 \%$ & 30 & $34.9 \%$ & 9 & $10.5 \%$ & 5 & $5.8 \%$ & $0.000^{*}$ \\
\hline Encouragement from mother & 11 & $8.3 \%$ & 121 & $91.7 \%$ & 0.292 & 63 & $47.7 \%$ & 33 & $25.0 \%$ & 12 & $9.1 \%$ & 24 & $18.2 \%$ & $0.007 *$ \\
\hline Social pressure on mothers & 8 & $21.1 \%$ & 30 & $78.9 \%$ & $0.008 *$ & 9 & $23.7 \%$ & 12 & $31.6 \%$ & 6 & $15.8 \%$ & 11 & $28.9 \%$ & $0.001^{*}$ \\
\hline $\begin{array}{l}\text { My personal } \\
\text { determinatior/experience }\end{array}$ & 14 & $9.7 \%$ & 130 & $90.3 \%$ & 0.273 & 66 & $45.8 \%$ & 42 & $29.2 \%$ & 12 & $8.3 \%$ & 24 & $16.7 \%$ & $0.028^{*}$ \\
\hline Husband encouraged me & 9 & $6.5 \%$ & 130 & $93.5 \%$ & $0.001 *$ & 66 & $47.5 \%$ & 42 & $30.2 \%$ & 12 & $8.6 \%$ & 19 & $13.7 \%$ & $0.005^{*}$ \\
\hline Nurse and midwife & 6 & $8.8 \%$ & 62 & $91.2 \%$ & 0.845 & 33 & $48.5 \%$ & 24 & $35.3 \%$ & 9 & $13.2 \%$ & 2 & $2.9 \%$ & $0.000^{*}$ \\
\hline $\begin{array}{l}\text { Encouragement from mother- } \\
\text { law }\end{array}$ & 8 & $8.0 \%$ & 92 & $92.0 \%$ & 0.435 & 54 & $54.0 \%$ & 21 & $21.0 \%$ & 6 & $6.0 \%$ & 19 & $19.0 \%$ & $0.014 *$ \\
\hline Media & 6 & $6.3 \%$ & 89 & $93.7 \%$ & 0.102 & 60 & $63.2 \%$ & 21 & $22.1 \%$ & 3 & $3.2 \%$ & 11 & $11.6 \%$ & $0.000^{*}$ \\
\hline My neighbours encouraged me & 5 & $8.9 \%$ & 51 & $91.1 \%$ & 0.895 & 36 & $64.3 \%$ & 15 & $26.8 \%$ & 3 & $5.4 \%$ & 2 & $3.6 \%$ & $0.001^{*}$ \\
\hline $\begin{array}{l}\text { Members of my religious } \\
\text { affiliation encouraged me }\end{array}$ & 9 & $13.2 \%$ & 59 & $86.8 \%$ & 0.135 & 36 & $52.9 \%$ & 18 & $26.5 \%$ & 6 & $8.8 \%$ & 8 & $11.8 \%$ & 0.518 \\
\hline $\begin{array}{l}\text { Baby continued to be hungry } \\
\text { after feeding }\end{array}$ & 5 & $4.9 \%$ & 97 & $95.1 \%$ & $0.009 *$ & 48 & $47.1 \%$ & 30 & $29.4 \%$ & 6 & $5.9 \%$ & 18 & $17.6 \%$ & 0.472 \\
\hline Matemal health problem & 0 & $0.0 \%$ & 11 & $100.0 \%$ & 0.134 & 3 & $27.3 \%$ & 3 & $27.3 \%$ & 0 & $0.0 \%$ & 5 & $45.5 \%$ & 0.057 \\
\hline $\begin{array}{l}\text { Fear of infant becoming } \\
\text { addicted to breast milk }\end{array}$ & 0 & $0.0 \%$ & 33 & $100.0 \%$ & $0.007 *$ & 24 & $72.7 \%$ & 9 & $27.3 \%$ & 0 & $0.0 \%$ & 0 & $0.0 \%$ & $0.000^{*}$ \\
\hline Due to pains in my breast & 6 & $14.3 \%$ & 36 & $85.7 \%$ & 0.210 & 30 & $71.4 \%$ & 3 & $7.1 \%$ & 3 & $7.1 \%$ & 6 & $14.3 \%$ & $0.000^{*}$ \\
\hline $\begin{array}{l}\text { was not making enough breast } \\
\text { milk to satisf my child }\end{array}$ & 5 & $4.2 \%$ & 115 & $95.8 \%$ & $0.000^{*}$ & 63 & $52.5 \%$ & 30 & $25.0 \%$ & 6 & $5.0 \%$ & 21 & $17.5 \%$ & $0.012 *$ \\
\hline I retumed to work/business & 14 & $100.0 \%$ & 0 & $0.0 \%$ & $0.000 *$ & 3 & $21.4 \%$ & 3 & $21.4 \%$ & 3 & $21.4 \%$ & 5 & $35.7 \%$ & $0.038^{*}$ \\
\hline Lack of husband's support & 2 & $11.8 \%$ & 15 & $88.2 \%$ & 0.723 & 12 & $70.6 \%$ & 3 & $17.6 \%$ & 0 & $0.0 \%$ & 2 & $11.8 \%$ & 0.124 \\
\hline
\end{tabular}


Citation: Ahmad Hasan M Alturkstani MD, MPH, Ahmed Mahmoud AlSaeed Alahdal MD, Sari Ibrahim Asiri MD, SBFM, Hossam Hussen Hijazy,2018 Barriers Of Exclusive Breastfeeding Among Mothers Attending Will -Baby Clinic In Phc, Makkah. Australian Journal of Basic and Applied Sciences., 12(1): 54-61.

\begin{tabular}{|l|l|l|l|l|l|l|l|l|l|l|l|l|l|l|} 
Breastfeeding was too tiring & 3 & $6.4 \%$ & 44 & $93.6 \%$ & 0.386 & 9 & $19.1 \%$ & 18 & $38.3 \%$ & 6 & $12.8 \%$ & 14 & $29.8 \%$ & $0.000^{*}$ \\
\hline $\begin{array}{l}\text { My neighbours pressured me to } \\
\text { wean the baby }\end{array}$ & 0 & $0.0 \%$ & 6 & $100.0 \%$ & 0.273 & 3 & $50.0 \%$ & 3 & $50.0 \%$ & 0 & $0.0 \%$ & 0 & $0.0 \%$ & 0.281 \\
\hline Baby refused breast milk & 0 & $0.0 \%$ & 39 & $100.0 \%$ & $0.003^{*}$ & 15 & $38.5 \%$ & 9 & $23.1 \%$ & 3 & $7.7 \%$ & 12 & $30.8 \%$ & $0.05^{*}$ \\
\hline was losing weight & 8 & $11.4 \%$ & 62 & $88.6 \%$ & 0.410 & 33 & $47.1 \%$ & 18 & $25.7 \%$ & 6 & $8.6 \%$ & 13 & $18.6 \%$ & 0.835 \\
\hline $\begin{array}{l}\text { I feel dizzy at times during } \\
\text { breastfeeding }\end{array}$ & 5 & $9.6 \%$ & 47 & $90.4 \%$ & 0.931 & 12 & $23.1 \%$ & 18 & $34.6 \%$ & 6 & $11.5 \%$ & 16 & $30.8 \%$ & $0.000^{*}$ \\
\hline Due to pregnancy & 5 & $35.7 \%$ & 9 & $64.3 \%$ & $0.003^{*}$ & 6 & $42.9 \%$ & 3 & $21.4 \%$ & 3 & $21.4 \%$ & 2 & $14.3 \%$ & 0.412 \\
\hline $\begin{array}{l}\text { My baby was not gaining } \\
\text { enough weight }\end{array}$ & 3 & $3.1 \%$ & 94 & $96.9 \%$ & $0.000^{*}$ & 45 & $46.4 \%$ & 24 & $24.7 \%$ & 9 & $9.3 \%$ & 19 & $19.6 \%$ & 0.245 \\
\hline I was not feeding well & 3 & $3.4 \%$ & 85 & $96.6 \%$ & $0.003^{*}$ & 36 & $40.9 \%$ & 30 & $34.1 \%$ & 6 & $6.8 \%$ & 16 & $18.2 \%$ & 0.104 \\
\hline
\end{tabular}

\section{Discussion:}

Breast milk is the safest and most natural food for an infant. It provides an infant's complete nutritional needs up to four to six months of age. There is no need for other food or drink before this age. When the baby is fed on breast milk only, it is called exclusive breastfeeding. Exclusive breastfeeding provides the best nutrition and growth for infants, and continued growth with the introduction of solid foods at six months breastfed during the first four months of life. (Amadhila, J.E., 2005)

The current study showed that the reasons for breast feeding preference is previous personal experience and encouragement from surrounding (husband, mother). They also consider factors that affecting the mother's preference and maintenance of exclusive breast feeding. Several studies reported that the experience of a mother, for example, experience of breastfeeding a previous child or not having any problems with breastfeeding during the first month, can be supportive to exclusive breast feeding. (Thepha et al., 2017)

The current study results revealed that, statistically significant differences were found between mothers' age, the exclusive breastfeeding. Younger mothers (20-25 years) were maintaining exclusive breastfed until 6 months of age. These results were supported by Woldie, et al., (2014) who mentioned that the women least likely to breastfeed are those who are young. Also this study agreed with Vogel et al., (2009) who revealed that, younger maternal age was associated with a short length of breastfeeding. The present study findings are dissimilar with the results of Gijsberset al., (2007) which shown that younger maternal age associated with a longer duration of breastfeeding. Women who are young may have low confidence in their ability to breastfeed and it is probable that older women know more about the benefits of breastfeeding and have more realistic outcome expectation. (Woldie, T., A. Kassa and M. Edris, 2014; Vogles, N., et al., 2009; Gijsbers, B., et al., 2007)

The majority of mothers mentioned that there was difficult to continue breast feeding with return to work and other women found it hard to maintain their milk supply when separated from their babies and were forced to stop breastfeeding. Working mothers were able to continue breastfeeding. The present study reveals statistically significant differences between the mothers' occupation and exclusive breast feeding and the mother return to work was a barrier for exclusive breast feeding. House wife mothers were more likely to exclusive breast fed their infants than working mothers; this finding was supported by Setegn et al., (2012) who showed that, unemployed mothers were about 5 times more likely to breastfed exclusively as compared to employed mothers. This may attribute to worked mothers were away from the home and from their infants which make the breast feeding difficult. (Setegn, T., et al., 2012; MacKean, G and W. Spragins)

In this study, the highest numbers of women were agree that the reasons for not exclusively breast fed were their infants were still feeling hungry after breastfed, feeling that the baby doesn't gain enough weight, perception of insufficient milk production, infants comfort and ease with formula feeding and breast milk dried up. In the same line, these findings were also in congruence with Negayama et al., (2012) \& Hawkins (2014) who clarified that the first or the second reason cited for initiating supplementation or weaning was perception of insufficient milk production. In addition Otsuka (2008) showed that 54\% mothers give an inadequacy of breast milk as reason for giving formula before six months. Also, Negayama (2012) mentioned that the most researchers found that approximately $35 \%$ of all women wean their children early, reporting that milk insufficiency was the primary reason. These findings may attribute to many women utilize infant satisfaction cues as their main indication of milk supply and many women do not evaluate actual milk supply. (Negayama, K., et al., 2012; Hawkins, S., et al., 2014; Otsuka, K., et al., 2008)

Barriers related to maternal factors identified in the review included being a teenage mother, working away from home, lack of support from surrounding and a lack of breastfeeding knowledge, which are similar to findings in TEKLE (2015). Additional barriers related to the mother were being a first time mother, her physical condition (such as nipple problems and breast pain) and negative attitudes to breastfeeding. Other studies have identified insufficient breast milk supply reported as a barrier to 6 months EBF by mothers. Family can be both a facilitator and a barrier. Support from family members (i.e. grandparents or the husband) was shown to have a positive impact on EBF, and this has been found in other studies. Lack of family support was also identified as barrier. (TEKLE, M. et al. 2015; Elsayed, H. et al., 2016)

Physical conditions in the mother, such as inverted, retracted or flat nipples, breast pain, postpartum depression, smoking and drug addiction were reported as influential barriers to EBF Moreover, mothers who had a caesarean section were more likely to report difficulty breastfeeding initially or "having to give formula milk". (Elsayed, H. et al., 2016)

\section{Conclusion:}

Based on the findings of the present study it can be concluded that the majority of mothers' housewife and multipara and not compliance with exclusive breast feeding. In addition the most common barriers to maintain exclusive breast feeding were the infants still hungry after feeding, perception of insufficient milk production, lack of support from surrounding and return to work. The study concluded that, there were many social constrains that influence mothers' exclusive breast feed their infants.

\section{Recommendations:}

- Efforts should be made in private and government hospitals, clinics, home-visits, to help educate the mothers about benefits of exclusive breast feeding.

- Encourage employed mothers to express breast milk for their exclusively breastfed infants and provide appropriate facilities and adequate time in the work place for breast feeding.

- Provide individualized education programme to mother and family members on health benefits of breastfeeding, and use culturally appropriate counseling techniques to explore knowledge, attitudes, beliefs, and personal ambivalence.

- Further research must be done to provide guidance towards preventing and overcoming common barriers to breastfeeding to the woman and her supportive family members during pregnancy.

Limitation of the study:

- Convenience sample.

- Small sample size.

\section{REFERENCES}

Froozani, M. D., Permehzadeh, K., Motlagh, A. R., \& Golestan, B. (1999). Effect of breastfeeding education on the feeding pattern and health of infants in their first 4 months in the Islamic Republic of Iran. Bulletin of the World Health Organization, 77(5), 381.

Otoo, G. E., Lartey, A. A., \& Pérez-Escamilla, R. (2009). Perceived incentives and barriers to exclusive breastfeeding among periurban Ghanaian women. Journal of Human Lactation, 25(1), 34-41. 
American Academy of Pediatrics, 2012. Breastfeeding and the use of human milk. Pediatrics, 129(3), e827e841. Available at http://dx.doi.org/10.1542/peds.2011-3552.

Thet, M. M., Khaing, E. E., Diamond-Smith, N., Sudhinaraset, M., Oo, S., \& Aung, T. (2016). Barriers to exclusive breastfeeding in the Ayeyarwaddy Region in Myanmar: Qualitative findings from mothers, grandmothers, and husbands. Appetite, 96, 62-69..

Mohamed, S., Zaki, N. A. E., \& Thabet, A. M., 2016. Barriers of Initiation and Exclusive Breast Feeding Among Infants IOSR Journal of Nursing and Health Science (IOSR-JNHS) e-ISSN: 2320-1959.p- ISSN: 2320-1940 5(2): 01-10.

Piper, S and P. Parks, 2012. "Predicting the duration of lactation: evidence from a national survey". Birth. 23: 7-12.

Danielle, W., J. Anneka, 2011. Female employees' perception of organizational support for breastfeeding at work: finding from Australian health service workplace. International Breastfeeding journal, 6: 19.

Agbo, H. A., Envuladu, E. A., Adams, H. S., Inalegwu, E., Okoh, E., Agba, A., \& Zoakah, A. I. (2013). Barriers and facilitators to the practice of exclusive breast feeding among working class mothers: a study of female resident doctors in tertiary health institutions in Plateau State. J Med Res, 2(1), 112-6..

Sibeko, L., Dhansay, M. A., Charlton, K. E., Johns, T., \& Gray-Donald, K. (2005). Beliefs, attitudes, and practices of breastfeeding mothers from a periurban community in South Africa. Journal of human lactation, 21(1), 31-38.

Kakute, P. N., Ngum, J., Mitchell, P., Kroll, K. A., Forgwei, G. W., Ngwang, L. K., \& Meyer, D. J. (2005). Cultural barriers to exclusive breastfeeding by mothers in a rural area of Cameroon, Africa. Journal of midwifery \& women's health, 50(4), 324-328.

Cohen, R. J., Brown, K. H., Rivera, L. L., \& Dewey, K. G. (1999). Promoting exclusive breastfeeding for 4-6 months in Honduras: attitudes of mothers and barriers to compliance. Journal of Human Lactation, 15(1), 9-18.

Amadhila, J.E., 2005. Factors that influence exclusive breast feeding in Windhoad district in Namibia. Published master thesis. University of Wastern Cape.

Woldie, T., A. Kassa and M. Edris, 2014. Assessment of exclusive breast feeding practice and associated factors in Mecha district, North west Ethiopia Science Journal of Public Health, 2(4): 330-336.

Vogles, N., D. Pothumus, E. Mariman, F. Bouwman, A. Kester, P. Rump, G. Honsta and M. Westerterp, 2009. Scientific opinion on the appropriate age for introduction of complementary feeding of infants. Determinants of overweight in a cohort of dutch children A.J.C.N. 84(4): 717-724.

Gijsbers, B., I. Mesters, J. knottnerus, C. VanSchayck, 2007. Factorsassociate d with the duration of exclusive breast feeding in asthmatic families. Health Educ, doi:10.1093/her/cyno13.

Setegn, T., T. Belachew, M. Gerbaba, K. Deribe, A. Deribew and S. Biadgilign, 2012. Factors associated with exclusive breastfeeding practices among mothers in Goba district, south east Ethiopia: a cross-sectional study.International Breastfeeding Journal, 7: 17.

MacKean, G and W. Spragins, The Challenges of Breastfeeding in a Complex World A critical review of the qualitative literature on women and their partners'/supporters' perceptions about breastfeeding.

Negayama, K., H. Norimatsu, M. Barratt and J. Bouville, 2012. Japan-France-US comparison of infant weaning from mother's viewpoint," Journal of Reproductive and Infant Psychology, 30(1): 77-91.

Hawkins, S., A. Stern, C. Baum and M, Gillman, 2014. Evaluating the impact of the Baby-Friendly Hospital Initiative on breast-feeding rates: a multi-state analysis," Public Health Nutrition, pp: 1-9.

Otsuka, K., C. Dennis, H. Tatsuoka and M. Jimba, 2008. The relationship between breastfeeding self-efficacy and perceived insufficient milk among Japanese mothers," Journal of Obstetric, Gynecologic\& ,Neonatal Nursing, 37(5): 546-555.

Tekle, M. T. (2015). Barriers to compliance to exclusive breastfeeding and timely introduction of complementary feeding practices in Ethiopia (Doctoral dissertation).

Nafee Elsayed, H. M., \& Al-Dossary, L. A. (2016). Exclusive Breastfeeding, Prevalence and Maternal Concerns: Saudi and Egyptian Mothers. Journal of Education and Practice, 7(3), 5-11.

\section{Highlights and contributions}

-Bacteria isolated from the natural environment can be used to produce economically important enzymes, using in agriculture, industrial and food fields.

-These bacterial isolates can grow and produce in a broad spectrum of salinity and high osmosis, can be used in biological treatments of various environmental wastes 